\title{
Sponsorship of overseas qualified doctors - exemption from the PLAB test
}

The General Medical Council has recently reviewed the arrangements for granting exemption from the PLAB test to overseas doctors. Although it will still be open to consultants to request limited registration with the General Medical Council for individual overseas qualified doctors whom they wish to train, these requests will in the future be made through the appropriate Royal College in the first instance.

The attention of members of the College is drawn to the following notice which originally appeared in the December 1993 issue of the General Medical Council News Review.

\section{Advice to consultants wishing to sponsor doctors from overseas for exemption from the PLAB test}

If you are planning to arrange for an overseas doctor to come to the United Kingdom for training and wish to obtain exemption from the PLAB test for him or her, you should approach the appropriate Royal College who will be able to guide you through the process of obtaining registration for the doctor.
Here is a quick check-list which may be helpful.

You should

seek advice from the appropriate Royal College about the procedures to be completed and the length of time this will take

initiate the process at least six months before the doctor arrives here

provide the Royal College with as much information as possible about the doctor's qualifications and experience: some primary medial qualifications granted overseas are not accepted by the GMC for any form of registration

be in a position to provide the Royal College with details of your knowledge of the doctor's sponsors overseas

ensure that the doctor is advised to bring to the United Kingdom the originals of his or her degree diplomas, medical college certificates (and marriage certificate, if relevant).

You should not

allow the doctor to take up employment until the GMC has confirmed the grant of limited registration.

\section{Continuing professional development workshops}

Introducing the first in the series of continuing professional development workshops to be offered by the College, this one-day workshop An Introductory Workshop on Stafi Supervision will be held on 5 September 1994 at The Royal College of Psychiatrists and is intended for both consultants and senior registrars. The aims of the workshop will be to offer an understanding of what constitutes effective supervision; to introduce a model of staff supervision; and to consider methods to enhance good supervisory practice.
Further workshops in the series include Ethics and Psychiatry: models and methods to be held on 4 October 1994 and Rationality and Culture: from hidden values to open agendas to be held on 12 October 1994. As numbers are limited early booking is advisable. Further information on these workshops and others in the series can be obtained from Annabel Thomas, CPD Department, The Royal College of Psychiatrists (telephone 071235 2351).

\section{CTC Reunion Dinner}

Unfortunately the Reunion Dinner planned for 6 July has had to be postponed.

The Dinner will now be held at the College on
Monday, 31 October 1994. Past members who are interested in attending, please contact Jean Wales at the College. 